CLINICAL STUDIES

\title{
Tympanic Versus Rectal Thermometry in Pregnant Women
}

SeonAe Yeo, RN, PhD, Robert H. Hayashi, MD, Jim Y. Wan, PhD, Barbara Dubler, RN, MSED, CNA

Objective: To assess the accuracy of the tympanic membrane thermometer for use with pregnant women.

Design: Cross-sectional descriptive study.

Setting: A major medical center in the midwestern United States. Participants: Thirty-three hospitalized, afebrile pregnant women.

Main outcome measures: Tympanic membrane thermometers and glass mercury thermometers were used to measure body temperature at the ear and rectum, respectively. The results were compared using two statistical methods: the Pearson correlation coefficient and a new technique suggested by Bland and Altman (1986).

Results: Auditory canal temperature measured by a tympanic membrane thermometer correlated with rectal temperature as measured by a glass mercury thermometer $(r=0.38, p=0.01)$. Thus, the tympanic membrane thermometer is acceptable for monitoring the body temperature of pregnant women. However, the device's estimation of rectal temperature is not clinically reliable.

Conclusions: Tympanic membrane thermometers, when applied with direct measures, are acceptable for use with pregnant women. It is not recommended that the rectal estimate mode be used with pregnant women.

Accepted: May 1994

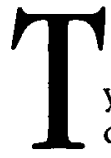

ympanic membrane thermometers (TMTs), also called infrared thermometers, have been gaining acceptance among health care professionals and the public. The TMT has some advantages over electronic oral and rectal thermometers. Major advantages are the TMT's speed of assessment, accuracy, and ease of placement. The TMT requires less than a second to measure body temperature with an infrared beam. Placement of the TMT in the auditory canal is easy because of the device's otoscope-like shape. Ease of placement and noninvasiveness make TMTs more comfortable for patients.
Nurses are less likely to be exposed to a patients' body secretions with a TMT than with an electronic oral or rectal thermometer. Because of the decreased chance of injury to the temperature site, nurses are less at risk of exposure to blood. Also, because TMTs involve no mercury, there is no chance of a mercury spill.

Tympanic membrane thermometers have been tested for their validity (Weiss, Pue, \& Smith, 1991), with children and nonpregnant adults (Chamberlain et al., 1991; Shenep et al., 1991; Talo, Macknin, \& Medendorp, 1991; Terndrup \& Milewski, 1991), and in different clinical settings (Milewski, Ferguson, \& Terndrup 1991; Selfridge \& Shea, 1993). The reliability of the TMT with nonpregnant populations has been tested by comparing tympanic and rectal temperatures (Selfridge \& Shea, 1993) and tympanic, axillary, oral, and rectal temperatures (Rogers et al., 1991). Newbold (1991) compared different brands. The TMT also has been tested for its repeatability (Terndrup \& Milewski, 1991), sensitivity (84\%) (Chamberlain et al., 1991; Shenep et al., 1991; Terndrup \& Milewski, 1991), and specificity (99\%) (Chamberlain et al., 1991; Shenep et al., 1991; Terndrup \& Milewski, 1991). Weiss et al. (1991) demonstrated the TMT's validity in detecting temperature. Reproducibility between left and right ear was found to range from $r=$ 0.89 to 0.92 (Weiss et al., 1991). Correlation coefficients between the auditory canal temperature and other body site temperatures, such as rectal, axillary, or pulmonary arteries, have demonstrated strong correlations, ranging from 0.77 to 0.98 (Heidenreich \& Giuffre, 1990; Shenep et al., 1991; Terndrup \& Milewski, 1991; Weiss et al., 1991). The above-cited studies concluded that TMTs are clinically acceptable. Nonetheless, what is acceptable with a nonpregnant population may not be acceptable with a pregnant population because of the physiologic adaptations that pregnancy imposes.

Maternal body temperature change has ramifications for both maternal and fetal well-being. Pregnancy alters a woman's temperature regulatory mechanism. This alteration makes the population of pregnant women different 


\section{Pregnancy alters a woman's temperature regulatory mechanisms.}

from other populations. Increased metabolic rate, in part caused by hormonal changes, produces more heat. A decrease in peripheral resistance allows an increased amount of blood to be shunted to the surface, dissipating more heat from the skin. The fetus must maintain a higher body temperature than the mother to dissipate the heat it produces. When a mother experiences hyperthermia because of an infection or an extremely hot environment, such as a sauna or a hot tub, fetal well-being is jeopardized in three ways: hypoxia secondary to maternal and fetal tachycardia and altered hemodynamics (Drinkwater \& Artal, 1991); teratogenesis (Edward, 1986; Miller, Smith, \& Shepard, 1978; Shiota, K., 1982); and preterm labor either from the fever, an underlying infection, or associated hemodynamic alterations (Blackburn \& Loper, 1992; Drinkwater \& Artal, 1991; Edward, 1986; Miller, Smith, \& Shepard, 1978; Shiota, 1982). Thus, it is critical for nurses to be able to measure maternal body temperature accurately.

\section{Rectal temperature is reported to correlate best with the pulmonary artery temperature, which is considered to be the most reliable indicator of core temperature.}

To test the accuracy of a new device, it must be compared with the current, standard, accepted device. Thus, the TMT must be compared with the glass mercury thermometer. Rectal temperature is reported to correlate best with pulmonary artery temperature, which is considered to be the most reliable indicator of core temperature (Heidenreich \& Giuffre, 1990). Heidenrich and Giuffre examined pulmonary artery, rectal, and axillary temperatures with 11 men and 7 women. They concluded that the correlation coefficient between rectal and core temperatures is $0.98^{\circ} \mathrm{C}$, and the difference between these two is $5^{\circ} \mathrm{C}$. Thus, if maternal tympanic and rectal temperatures correlate highly and if the difference between the two measures is stable, the TMT could be accepted as a reliable thermometer for use with pregnant women.

The purpose of the current study was to compare the TMT with the glass mercury thermometer for use with pregnant women. The new device will be considered acceptable if the variance of the difference between the two measures is narrow enough (i.e., a standard deviation of less than $0.5^{\circ} \mathrm{C}$ ) and the correlation between the two measures is statistically strong $(p<0.01)$. In addition, the accuracy of one of the features of the TMT, estimation of rectal temperature, will be assessed for pregnant women.

\section{Metbods}

\section{Subjects}

The current study assessed the clinical applicability of the TMT with a cross-section of pregnant women. A convenience sample was chosen from pregnant women who were hospitalized at a major medical center in the midwestern United States between August and December 1992. The sample consisted of 33 pregnant women, at various stages of gestation ( $M=24$ weeks; $S D=1$ week), who were not receiving prostaglandin treatment and who were not experiencing uterine contractions. The study was approved by the institution's human subjects review committee.

\section{Variables}

The variables were the rectal temperature measured by a glass mercury thermometer, the auditory canal temperature measured by a TMT, and the estimated rectal temperature extrapolated by a TMT from the auditory canal temperature.

The Thermoscan Pro-1 (Thermoscan Inc., San Diego, CA) was used for the study. A small computer installed in the device provides estimates of other body site temperatures, based on a thermal model developed by Fraden and Lackey (1991). Thus, the TMT placed at the ear provides estimates of rectal temperature. According to Fraden and Lackey (1991),

1. Temperatures of interest (e.g., core or tympanic) are considered to be localized at specific points rather than distributed over a surface.

2. Thermal resistance between specific points is linear. Such resistance represents the combined effects of heat movement resulting from blood circulation, heat conduction, air convection, and infrared radiation.

3. The core, mouth, and rectum are thermally disconnected (thermal resistance between them is infinitely large); however, they are under common physiologic control (p. 67.)

On the basis of the above assumptions, the model equation was developed as follows:

$$
\mathrm{Tc}=\mathrm{Ta}+(\mathrm{Tb}-\mathrm{Ta})(1+\text { beta })
$$

Tc is a hypothetical core temperature, Ta is an ambient temperature, $\mathrm{Tb}$ is an auditory canal temperature, and beta is an environment-to-core coupling coefficient (Fraden $\&$ Lackey, 1991). With the above equation, one can estimate a hypothetical core temperature by knowing the ear temperature, the room temperature, and the beta. According to Fraden and Lackey, one can calculate betas for rectum, oral, or other body locations with the above 
equation, replacing Tc with the corresponding body site temperature. Thus, the beta for rectal temperature is as follows:

$$
\text { beta }=\frac{(T r-T b)}{(T b-T a)}
$$

$\mathrm{Tr}$ is the rectal temperature, $\mathrm{Tb}$ is the ear temperature, and $\mathrm{Ta}$ is the ambient temperature. Fraden and Lackey calculated and installed the beta into the TMT for the rectal temperature estimate based on data from children younger than 3 years of age. Fraden and Lackey (1991) determined the beta for rectal estimate to be 0.081 . This coupling coefficient is installed in the TMT. Glass mercury thermometers without probe covers (Bax. ter Health Care, Deerfield, IL) were used for the study.

\section{Procedures}

A TMT and a glass mercury thermometer were used to measure body temperature at regular vital sign assessments. After screening patients according to the study criteria and before data collection, patients were asked if they had been within the building less than 1 hour and, therefore, were not acclimated to the ambient temperature; if they had engaged in vigorous physical activity within the past hour (walking beyond their room was considered vigorous activity); and if they were experiencing any untoward emotions (e.g., anger or depression). If patients answered yes to one or more of the questions, data collection was postponed until the conditions were resolved. Documentation of the above conditions is important because they can have an immediate effect on tympanic membrane temperature, whereas rectal temperature corresponds more slowly (Fraden \& Lackey, 1991).

Rectal temperature was measured with a new glass mercury thermometer at each assessment. A glass mercury thermometer was inserted $1 \frac{1}{2}$ inches into the rectum for 1 minute (Nichols, Kucha, \& Mahoney, 1972). The 1-minute placement time was chosen on the basis of Nichols's report that a 1-minute placement time for rectal temperature with afebrile adults will reach the lowest acceptable percentage $(80 \%)$ of optimal temperature (Nichols, Kucha, \& Mahoney 1972). After the rectal temperature measure, the TMT was applied to the auditory canal with a disposable probe cover. Subjects were asked to hold their heads still. To get an accurate reading, the ear canal was straightened by tugging the ear as for a standard otoscopic examination. The probe was aimed slightly forward of the opposite ear. A slight rocking motion was used to insert the probe as far as possible and to seal the ear canal. Either the left or the right ear was chosen, but the necessary two measurements (i.e., one for the direct reading and another for the estimate for the rectal temperature based on the thermal model) were performed on the same side.

\section{Statistical Analyses}

Pearson correlation coefficients were calculated for the rectal temperature as measured by a glass mercury ther-
Table 1. Mean Body Temperatures at Different Body Sites

\begin{tabular}{llll}
\hline Instrument & $\mathrm{N}$ & $\mathrm{M}$ & $\mathrm{SD}$ \\
\hline Glass mercury thermometer & & & \\
$\quad$ Rectal & 33 & 37.4 & 0.36 \\
Tympanic membrane thermometer & & & \\
$\quad$ Auditory canal & 33 & 36.7 & 0.37 \\
$\quad$ Rectal estimate & 30 & 37.6 & 0.34 \\
\hline
\end{tabular}

mometer and for the auditory canal temperature as measured by a TMT. This is the standard accepted method of assessing the reliability of two tools (Bland \& Altman, 1986). The disadvantage of this approach is that neither the established nor the new measure provides true val. ues, which can be assessed by means of calibration in most clinical settings. Thus, even if the correlation coefficient is significant, the difference in two measured values may vary more than an acceptable level.

Bland and Altman (1986) reported a new statistical method for assessing agreement between two methods of clinical measurement. They maintain that the use of correlation is misleading for a clinical comparison of a new measurement technique with an established one, and they suggest a new approach based on graphic techniques and simple calculations. Using this new approach, investigators plot the mean of rectal and auditory canal temperature on the $x$-axis and the difference of these values on the $y$-axis. Each data point indicates mean temperature between rectal and auditory canal temperature on the horizontal line and the difference between rectal and auditory canal temperature on the vertical line. The graphic of the result (see Figure 1) indicates whether the discrepancy between the two measurements is associated with the range of raw data. Investigators assess the degree of acceptability graphically. Bland and Altman's (1986) technique seems to complement the traditional method-the Pearson correlation coefficient. In the current study, Bland and Altman's approach and the traditional Pearson's correlation coefficient were used.

The coupling coefficient based on Fraden and Lackey's thermal model was estimated for a population of pregnant women. This process allowed assessment of whether the installed coupling coefficient in the product, Thermoscan Pro-1 (Thermoscan Inc.), was valid for a population of pregnant women. The coupling coefficient of 0.081 was used for the product to estimate the rectal temperature.

\section{Results}

Table 1 shows the means of each variable. The mean of the rectal temperature measured by a glass mercury thermometer was higher than the mean of the auditory canal temperature measured by a TMT by $0.7^{\circ} \mathrm{C}$. The mean of the estimated rectal temperature extrapolated by a TMT from the auditory canal temperature was higher than the rectal temperature measured by a glass mercury ther- 
Table 2. Correlation and Mean Difference Between Measurements

\begin{tabular}{lcccc}
\hline Instrument & $\begin{array}{l}\text { Pearson Correlation } \\
\text { Coefficient } \mathrm{r}(\mathrm{p})\end{array}$ & $\begin{array}{l}\text { Mean Difference }\left({ }^{\circ} \mathrm{C}\right) \\
\mathrm{M}\left(\mathrm{SD}^{*}\right)\left(\mathrm{SEM}^{*}\right)\end{array}$ & $\begin{array}{r}\text { Limits of Agreement }\left({ }^{\circ} \mathrm{C}\right) \\
\text { 95\% Confidence Interval }\end{array}$ & $\begin{array}{r}\mathrm{N} \\
\text { GMR and I }\end{array}$ \\
GMR and ERI & $0.38(0.01)$ & $0.67(0.40)(0.07)$ & 33 & $0.53 \sim 0.81$ \\
ERI and I & $18(0.18)$ & $-0.26(0.45)(0.08)$ & 30 & $-0.42 \sim-0.1$ \\
\end{tabular}

Note. SEM = Standard error of measurement; GMR = rectal temperature measured by a glass mercury thermometer; $I=$ auditory canal temperature measured by tympanic membrane thermometer; ERI = estimated rectal temperature from I.

mometer by $0.2^{\circ} \mathrm{C}$. Although small, the difference $\left(0.2^{\circ} \mathrm{C}\right)$ between the means is significant $(t=3.19, p=$ $0.003)$.

Table 2 indicates Pearson correlation coefficients between variables. The correlation coefficient between the rectal temperature measured by a glass mercury thermometer and the auditory canal temperature measured by a TMT is significant, yet relatively low $(r=0.38, p=$ 0.01 ). This small correlation may be attributed to a narrow range of data distribution compared with the distribution of the standard deviation, in addition to an intended clinically realistic data collection condition (i.e., 1 minute of rectal temperature measurement time). Agreement between the auditory canal temperature measured by a TMT and the estimated rectal temperature extrapolated by a TMT from the auditory canal temperature was, as expected, stronger. The correlation coefficient was $0.78(p<0.0001)$, the mean difference was $0.92^{\circ} \mathrm{C}$, and the standard deviation was $0.23^{\circ} \mathrm{C}$.

Agreement between the rectal temperature measured by a glass mercury thermometer and the estimated rectal temperature extrapolated by a TMT from the auditory canal temperature is depicted in Figure 1A. This graph shows the difference between the two measurements against the mean of the two measurements. The majority of the data indicate that the differences between the two measures are distributed within 1 standard deviation $\left( \pm 0.45^{\circ} \mathrm{C}\right)$ of the mean difference $\left(-0.26^{\circ} \mathrm{C}\right)$.

Agreement between the rectal temperature measured by a glass mercury thermometer and the auditory canal temperature measured by a TMT is shown in Figure 1B. Most of the data indicate that the differences between the two measures are distributed within 1 standard deviation $\left( \pm 0.4^{\circ} \mathrm{C}\right)$ of the mean difference $\left(0.67^{\circ} \mathrm{C}\right)$. The pattern of distribution demonstrates a horizontal belt, sug. gesting there is no underlying measurement influence. (Measurement influence means that the difference of the two measures depends on raw data.)

The mean of the coupling coefficient for rectal temperature with the sample group is 0.049 , and the standard error of the estimate is 0.005 . Accordingly, the $95 \%$ confidence interval is $0.049 \pm 0.0098$ (i.e., 0.039 and 0.059 ). The currently installed coupling coefficient in the TMT is 0.081 . Thus, the use of the estimated rectal temperature extrapolated by a TMT from the auditory canal temperature is not appropriate for pregnant women.

\section{Discussion}

The current study sheds light on the issue of the relationship between body site temperatures and pregnant women. Despite the relatively low, yet significant, corre-
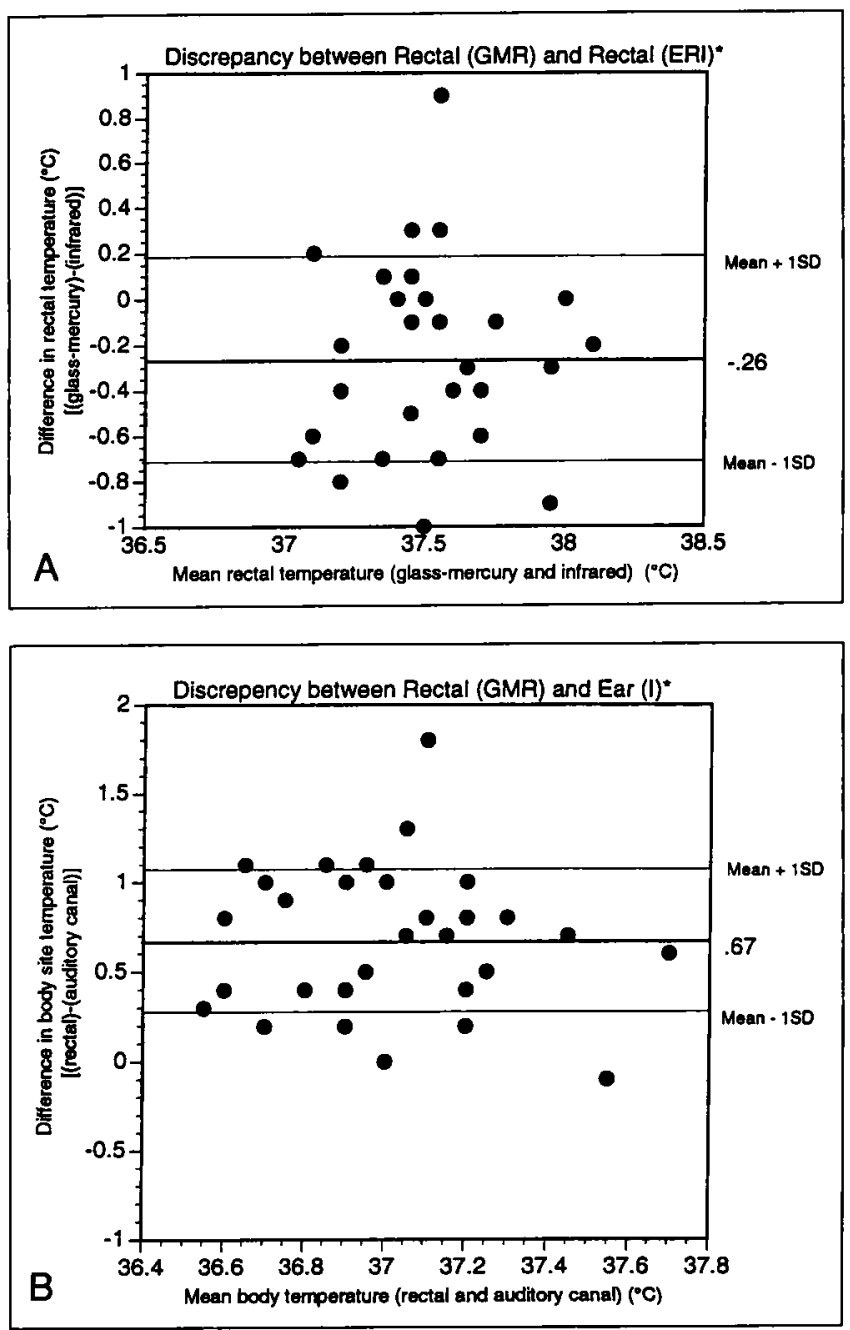

Figure 1. Plots of $(A)$ direct infrared thermometry and $(B)$ indirect infrared thermometry against glass mercury rectal thermometry. relating the discrepancy between the two measuring systems. GMR = glass mercury thermometry: I and $E R I=$ infrared thermometry. 
lation coefficient, infrared thermometers are acceptable for use with pregnant women because of reasonable agreement between the new measurement and an established measurement (i.e., the glass mercury thermometer). Other issues, however, may merit consideration in deciding whether to replace glass mercury thermometers with TMTs.

\section{More women are experiencing increasingly early discharge from hospitals. During this critical time for both mother and newborn, any temperature elevation merits close monitoring.}

The cost effectiveness of TMTs is better than or similar to electronic oral or rectal thermometers. The price of the TMTs for professional use varies depending on brand, ranging from $\$ 300$ to $\$ 378$ per unit. Ease and speed of use of the TMTs may save nurses' time. Implications of the use of the TMT are not limited to the antepartum period. Physiologic modifications during the intrapartum and postpartum periods are more dramatic. Within the first 24 hours of delivery, transient maternal temperature elevations as high as $38^{\circ} \mathrm{C}$ occur in as many as $6.5 \%$ of women who have given birth vaginally (Blackburn \& Loper, 1991). The majority of transient hyperthermia resolves spontaneously because it is secondary to dehydration or to transient bacterial endometritis. Yet, the possibility of puerperal infection, mastitis, endometritis, or urinary tract infection cannot be negated (Blackburn \& Loper, 1991). More women are experiencing increasingly early discharge from hospitals after childbirth. During this critical time for both mother and newborn, any temperature elevation merits close monitoring. At one third the cost of the professional model, the home version of TMT may be a worthwhile investment for women.

An additional benefit of advocating TMTs for use outside the clinical setting may be found in monitoring the body temperature of exercising pregnant women. Because of its speed and safety, a TMT may be used to monitor a pregnant woman's body temperature during exercise. The American College of Obstetricians and Gynecologists (ACOG) recommends that maternal body temperature be maintained no higher than $38^{\circ} \mathrm{C}$ (ACOG, 1985). Yet, except with TMTs, it is almost impossible to measure body temperature during or immediately after exercise. Further research is needed, however, to examine the safe range of the auditory canal temperature for pregnant women during exercise because ACOG's guideline is not provided specifically for auditory canal temperature.

These benefits notwithstanding, the limitations of the current study and TMTs for obstetric nursing must be addressed. First, it is not recommended that rectal temperature be estimated with TMTs. The mean difference between glass mercury thermometry of the rectum and estimation of rectal temperature by a TMT remains small but significant $\left(0.26^{\circ} \mathrm{C}\right)$; TMTs overestimate the rectal temperature of pregnant women.

The direction of the difference between rectal and auditory canal temperatures (i.e., rectal temperature greater than auditory canal temperature) in the current study is consistent with others' findings (Milewski et al., 1991; Talo et al., 1991), but the magnitude of difference is larger in the above-cited studies. Talo et al. (1991) reported the mean ear-rectal difference as $1.1^{\circ} \mathrm{C}$, whereas Milewski et al. (1991) reported the difference as $1.2^{\circ} \mathrm{C}$. The current study indicates the mean ear-rectal difference as $0.67^{\circ} \mathrm{C}$. It is unknown whether these differences are the result of population disparity or are unique to study designs. For example, the Talo et al. study involved a sample of children who were most likely febrile, and the Milewski et al. study involved a sample of nine adult patients in intensive-care units. The current study used a sample of afebrile pregnant women at a hospital.

Questions remain with regard to the measurement of body temperature during pregnancy:

What body site should be referred to for pregnant women's body temperature, considering the wellbeing of both mother and fetus?

What is normal body temperature, and is it a function of gestation? Clapp, Wesley, and Sleamaker (1987) reported that the resting body temperature of pregnant women increases throughout the 1st trimester and remains constant afterward, whereas the current authors' unpublished preliminary data indicate that resting temperature decreases during the 1 st trimester and increases thereafter.

How does maternal body site temperature relate to fetal temperature? This last issue may have implications during prolonged labor or during exercise.

Tympanic membrane thermometers are acceptable for use with pregnant women because TMT measurement takes less time than does measurement with other thermometers and because TMT measurements reasonably agree with glass mercury thermometer measurements. However, it is advisable to use the direct measurement mode of the TMT.

\section{References}

American College of Obstetricians and Gynecologists. (1985). Home exercise programs. Washington, DC: Author.

Blackburn, S. T., \& Loper, D. L. (1992). Maternal, fetal, and neonatal physiology: A clinical perspective. Philadelphia: W.B. Saunders.

Bland, J. M., \& Altman, D. G. (1986). Statistical methods for assessing agreement between two methods of clinical mea surement. Lancet, 1, 307-310.

Chamberlain, J. M., Grandner, J. G., Rubinoff, J. L., Klein, B. L., 
Waisman, Y., \& Huey, M. (1991). Comparison of a tympanic thermometer to rectal and oral thermometers in a pediatric emergency department. Clinical Pediatrics, 30(Suppl. 4), 25-29.

Clapp, J. F. III, Wesley, M., \& Sleamaker, R. H. (1987). Thermoregulatory and metabolic responses to jogging prior to and during pregnancy. Medicine and Science in Sports and Ex. ercise, 19, 124-130.

Drinkwater, B. L., \& Artal, R. M. (1991). Heat stress and pregnancy. In R. M. Artal, R. A. Wiswell, \& B. L. Drinkwater (Eds.), Exercise in pregnancy (2nd ed.) (pp. 261-270). Baltimore: Williams \& Wilkins.

Edward, M. J. (1986). Hyperthermia as a teratogen. Teratologen and Carcinogenic Mutagen, 6, 563-582.

Fraden, J., \& Lackey, R. P. (1991). Estimation of body sites temperatures from tympanic measurements. Clinical Pediatrics, 30(Suppl. 4), 65-70.

Heidenreich, T., \& Giuffre, M. (1990). Postoperative temperature measurement. Nursing Research, 39, 153-155.

Milewski, A., Ferguson, K. L., \& Terndrup, T. E. (1991). Comparison of pulmonary artery, rectal, and tympanic membrane temperatures in adult intensive care unit patients. Clinical Pediatrics, 30(Suppl. 4), 13-16.

Miller, P., Smith, D. W., \& Shepard, T. H. (1978). Maternal hyperthermia as a possible cause of anencephaly. Lancet, 1 , 519-521

Morishima, H., Yeh, M., Niemann, W. H., \& James, L. S. (1977). Temperature gradient between fetus and mother as an index for assessing intrauterine fetal condition. American Journal of Obstetrics and Gynecology, 129, 443-448.

Newbold, J. (1991). Evaluation of a new infrared tympanic thermometer: A comparison of three brands. Journal of Pediatric Nursing, G(4), 281-283.

Nichols, G. A., Kucha, D. H., \& Mahoney, R. P. (1972). Rectal thermometer placement times for febrile adults. Nursing Research, 21(1), 76-77.

Rogers, J., Curley, M., Driscoll, J., Kerrigan, T., LeBlanc, G., Libman, M., \& McCarty, K. (1991). Evaluation of tympanic membrane thermometer for use with pediatric patients. $P e$. diatric Nursing, 17(4), 376-378.

Selfridge, J., \& Shea, S. S. (1993). The accuracy of the tympanic membrane thermometer in detecting fever in infants aged
3 months and younger in the emergency department setting. Journal of Emergency Nursing, 19(2), 127-130.

Shenep, J. L., Adair, J. R., Hughes, W. T., Roberson, P. K., Flynn, P. M., Brodkey, T. O., Fuller, G. H., Kennedy, W. T., Oakes, L. L., \& Marina, N. M. (1991). Infrared thermistor and glass mercury thermometry for measurement of body temperature in children with cancer. Clinical Pediatrics, 30 (Suppl. 4), 36-41

Shiota, K. (1982). Neural tube defects and maternal hyperthermia in early pregnancy: Epistemology in human embryonic population. American Journal of Medical Genetics, 12, 281-288.

Talo, H., Macknin, M. L., \& Medendorp, S. V. (1991). Tympanic membrane temperature compared to rectal and oral temperature. Clinical Pediatrics, 30(Suppl. 4), 30-33.

Terndrup, T. E., \& Milewski, A. (1991). The performance of two tympanic thermometers in a pediatric emergency depart ment. Clinical Pediatrics, 30(Suppl. 4), 18-23.

Weiss, M. E., Pue, A. F., \& Smith, J., III. (1991). Laboratory and hospital testing of new infrared tympanic thermometers. Journal of Clinical Engineering, 16(2), 137-144.

Address for correspondence: SeonAe Yeo, RN, $P b D$, University of Michigan School of Nursing, 400 N. Ingalls, Ann Arbor, MI 48109. 0482.

SeonAe Yeo is an assistant professor in the Diviston of Health Promotion and Risk Reduction at the Scbool of Nursing, University of Michigan, Ann Arbor.

Robert H. Hayasht is a professor and the Director of Maternal-Fetal Medicine, Medical School, University of Michigan.

Jim Y. Wan is an assistant professor in the Department of Biostatistics, University of Tennessee, Memphis.

Barbara Dubler is a bead nurse in the Antepartum Untt of the University of Michigan Medical Center, Ann Arbor.

\section{Notice to Copiers}

Authorization to photocopy items for internal or personal use, or the internal or personal use of specific clients, is granted by AWHONN, the Association of Women's Health, Obstetric, and Neonatal Nurses for libraries and other users registered with the Copyright Clearance Center (CCC), provided that the base fee of $\$ 10$ per copy is paid directly to CCC, 222 Rosewood Drive, Danvers, MA 01923. 0884-2175/95 \$10. The U. S. Government retains a nonexclusive, royalty-free license in and to any copyright on articles authored by government employees. 